\title{
A Study of Xylene Free Hematoxylin and Eosin Staining Procedure
}

\author{
Anikode Subramanian Ramaswamy* and Panati Dayasagar
}

Department Of Pathology, P E S Institute Of Medical Sciences And Research, Kuppam, Chittoor, Andhra Pradesh, India

\section{ABSTRACT}

Background: Mineral oils and liquid dish washing solutions have been tried by various investigators as a substitute for xylene in order to eliminate or minimize its toxic and hazardous effects.

Methods: One hundred paraffin embedded sections each were stained by both conventional method and xylene free method using liquid dish washer. The sections were scored on the parameters of nuclear staining, cytoplasmic staining, crispness of staining, uniformity of staining and clarity of staining. The results of the two were compared and statistically analyzed.

Result: The statistical analysis suggests that the soapy sections failed to prove that it has comparable effects as that of the conventional method.

Conclusion: Liquid dish washer can be a safer non toxic alternative to xylene. More studies using modifications of the above technique can be undertaken to improve the quality of stained sections thus eliminating occupational hazards.

\section{Keywords: Xylene, Hematoxylin, Detergents, Occupational Exposure, Histological Techniques}

\section{Introduction}

The diagnostic armamentarium of a pathologist includes histopathological diagnosis of various lesions by the examination of biopsy tissues submitted. The study includes various steps like fixation, dehydration, clearing, impregnation, embedding, sectioning and staining. The most commonly used method of processing is the formalin fixed, paraffin embedded method with the sections being stained by Haematoxylin and Eosin (H\&E) stains. H\&E remains the most common and most popular of the stains. It provides a pleasing color to the eyes of the examining pathologist with optimal contrast. ${ }^{[1]}$

The aforementioned schedule of tissue processing, sectioning and staining entails the use of various chemicals like formalin, xylene and alcohol. Many of these chemicals are known to be irritants, toxic and also carcinogenic. They are also environmentally unfriendly to dispose. ${ }^{[2,3,4]}$ Xylene and alcohol are commonly used in the H\&E staining procedure for the process of dewaxing, clearing and dehydration. ${ }^{[1]}$ The exposure of the histotechnician to the toxic chemicals like xylene and alcohol is maximum during the process of H\&E staining and efforts are on to reduce this occupational hazard..$^{[1,2,3,4]}$

Xylene is a chemical which is widely used in various industries ranging from rubber to leather industry. In the field of histopathology, it is primarily employed as a clearing agent during tissue processing of biopsies and also during staining of sections. Its high solvency factor allows maximum displacement of alcohol. This makes the tissue more transparent which enhances infiltration by paraffin. In staining of sections, it has superior dewaxing capacity combined with clearing capability. This makes the sections to appear pleasing to visualize. ${ }^{[3,4]}$

Laboratory grade xylene is composed of m-xylene, p-xylene and o-xylene along with ethyl benzene, traces of toluene, trimethyl benzene, phenol, thiphene, pyridine and hydrogen sulfide. Histopathological technicians by virtue of the nature of their work are maximally exposed to xylene. The current Occupational Safety and Health Administration permissible exposure limit for xylene is $100 \mathrm{ppm}$ as an 8 hour time weighted average (TWA) concentration. The National Institute for Occupational Safety and Health recommended exposure limits for xylene at $100 \mathrm{ppm}$ as a TWA for up to a 10 hour work shift and a 40 hour week and 200ppm for 10 min as a short term limit. ${ }^{[2,3,4]}$

Apart from being a cause for occupational exposure, unsafe disposal can lead to human contact via soil or water contamination where it may remain for months before it breaks down into other chemicals. ${ }^{[3,4]}$

A person can get exposed to xylene via inhalation, ingestion, eye or dermal contact. It is primarily metabolized in the liver and excreted in the urine with smaller amounts eliminated unchanged in exhaled air. The health effects of xylene can be both due to acute ( $<14$ days) and also 
chronic ( $>365$ days) exposure. As expected, a technologist in the histopathology laboratory can have both the types of exposure. The harmful effects of xylene are practically seen in every organ system ranging from depression of the central nervous system to contact dermatitis unless proper personal protection is taken care of. Various alternatives have been tried to eliminate these toxic substances which are helpful both to the technician and to the environment. ${ }^{[5-}$ ${ }^{9]}$ These methods have met with varying degree of success.

Preventive measures include substitution, local exhaust ventilation and use of proper protective equipments. Of these, substitution is the most promising method with various substances being tried that can perform the same function with lesser or no hazard as xylene.

Limonene reagents, aliphatic hydrocarbon mixtures, aromatic hydrocarbon mixtures and mineral oil mixtures have been tried as substitutes for xylene. ${ }^{[5,9]}$

A relatively non toxic, non irritant substance which has been less frequently studied has been the liquid detergent commercially available in shampoos and dishwashing solutions. The ease of availability of these detergents with its non toxic nature makes it an attractive and cost effective alternative to xylene. ${ }^{[5,6,7,10]}$ The present study intends to utilize the commercially available liquid dishwashing solution as a substitute for xylene and alcohol in dewaxing and staining.

\section{Aims and Objectives}

1. To test the hypothesis that sections deparaffinised with diluted liquid dishwasher (LDW) solution are better than xylene and alcohol treated H\&E stained sections

2. To compare the quality of dishwasher solution treated $H \& E$ sections with that of xylene and alcohol treated H\&E sections

\section{Materials and Methods}

The present prospective study was carried out in the Department of Pathology over a period of 2 months between May and June 2015.

One hundred consecutively prepared formalin fixed, paraffin embedded tissue blocks of various surgical specimen submitted for histopathological examination in this period were selected. Two sections of $4 \mu \mathrm{m}$ thickness were cut from each block. One of the sections was processed by the conventional method of $\mathrm{H} \& \mathrm{E}$ staining as follows

1. Xylene 1- 5 minutes

2. Xylene 2- 5 minutes

3. $90 \%$ alcohol -5 minutes

4. $70 \%$ alcohol -5 minutes
5. Water wash -10 minutes

6. Harri's hematoxylin -6 minutes

7. Water wash -2 minutes

8. $1 \%$ acid alcohol -1 dip

9. Water wash -10 minutes

10. $1 \%$ lithium carbonate -1 minute

11. Water wash -10 minutes

12. $1 \%$ eosin -1 minute

13. $90 \%$ alcohol -1 minute

14. $70 \%$ alcohol -1 minute

15. Xylene 1- 5 minutes

16. Xylene 2- 5 minutes

17. Mount with DPX and cover slip

The other section was stained using liquid dishwasher solution as follows. Commercially available $\mathrm{Vim}^{\mathrm{TM}}$ liquid dishwasher solution was procured. To $1000 \mathrm{ml}$ of distilled water $25 \mathrm{ml}$ of the liquid dishwasher (LDW) was added and the diluted mixture was used for staining as follows.

1. Diluted LDW 1- at $90{ }^{\circ} \mathrm{C} 1$ minute

2. Diluted LDW 2- at $90{ }^{\circ} \mathrm{C} 1$ minute

3. Distilled water - at $90{ }^{\circ} \mathrm{C} 30$ seconds

4. Distilled water - at $90{ }^{\circ} \mathrm{C} 30$ seconds

5. Distilled Water wash - at $45{ }^{\circ} \mathrm{C} 10$ minutes

6. Harri's haematoxylin -6 minutes

7. Water wash -2 minutes

8. $1 \%$ acid alcohol -1 dip

9. Water wash -10 minutes

10. $1 \%$ lithium carbonate -1 minute

11. Water wash -10 minutes

12. $1 \%$ eosin -1 minute

13. Water wash -1 minute

14. Oven dry the sections at $60{ }^{\circ} \mathrm{C}$ for 10 minutes

15. Mount with DPX and cover slip

The sections were then coded as $\mathrm{C}$ for the conventional and L for the LDW treated methods. The sections were then analyzed by a single pathologist who was blinded to the treatment protocols. The H\&E sections were then scored as follows

1 Nuclear staining: adequate (score 1), inadequate (score 0)

2. Cytoplasmic staining: adequate (score 1), inadequate (score 0) 
3. Clarity of staining: present (score 1), absent (score 0)

4. Uniformity of staining: present (score 1), absent (score 0)

5. Crispness of staining: present (score 1), absent (score 0)

A score of 0-1 was assigned for each parameter. The total scores were added and a score $</=2$ was considered as inadequate for diagnosis and scores 3-5 as adequate for diagnosis. Test for proportions was used for comparing the two staining methods and a $\mathrm{P}$ value $<0.05$ was considered as significant.

\section{Result}

After application of scores to the individual sections by the pathologist the following scores were noted as depicted in the table 1 . As noted, the nuclear staining was adequate in both the methods. The parameter of cytoplasmic staining too in the LDW method was almost the same as that of the conventional method. The LDW method scored fairly on the attributes of clarity of staining and crispness of staining. However, the LDW treated sections did not show uniformity in staining when compared to the conventional method.

All the 100 cases processed by the conventional method were adequate for diagnosis. In the LDW method 84 out of 100 sections scored in the range of 3-5 and were considered adequate for diagnosis.

Proportions test for comparing the two staining methods showed that there is a statistical difference between the two proportions. It suggests that the soapy sections failed to prove that it has comparable effects as that of the conventional method. The $\mathrm{p}$ value obtained was $<0.001$.

Table 1: Comparitive Scores Obtained in the Conventional and LDW Staining Protocols.

\begin{tabular}{|l|c|c|c|c|}
\hline Parameter & Conventional Adequate & Conventional Inadequate & LDW Adequate & LDW Inadequate \\
\hline Nuclear staining & 100 & 0 & 100 & 0 \\
\hline Cytoplasmic staining & 100 & 0 & 95 & 05 \\
\hline Clarity of staining & 100 & 0 & 75 & 25 \\
\hline Uniformity of staining & 98 & 02 & 07 & 93 \\
\hline Crispness of staining & 100 & 0 & 77 & 23 \\
\hline
\end{tabular}

Table 2: Comparitive Table Depicting Scores Obtained in Different Studies.

\begin{tabular}{|c|c|c|c|c|c|c|c|c|c|c|c|c|c|c|c|c|c|c|c|c|c|c|c|c|c|}
\hline & \multicolumn{4}{|c|}{ Nuclear staining } & \multicolumn{4}{|c|}{$\begin{array}{l}\text { Cytoplasmic } \\
\text { staining }\end{array}$} & \multicolumn{4}{|c|}{$\begin{array}{l}\text { Clarity of } \\
\text { staining }\end{array}$} & \multicolumn{4}{|c|}{$\begin{array}{l}\text { Uniformity of } \\
\text { staining }\end{array}$} & \multicolumn{4}{|c|}{$\begin{array}{l}\text { Crispness of } \\
\text { staining }\end{array}$} & \multicolumn{4}{|c|}{$\begin{array}{l}\text { Scores for } \\
\text { adequacy for } \\
\text { diagnosis }\end{array}$} & \multirow[t]{2}{*}{$\begin{array}{c}\text { Total } \\
\text { number } \\
\text { of cases }\end{array}$} \\
\hline & $A$ & B & C & $\mathrm{D}$ & $A$ & B & C & $\mathrm{D}$ & A & B & C & $\mathrm{D}$ & $A$ & $B$ & C & D & A & B & C & $\mathrm{D}$ & $A$ & $B$ & C & $D$ & \\
\hline Ank & 58 & 02 & 59 & 01 & 56 & 04 & 50 & 10 & 51 & 09 & 53 & 07 & 42 & 18 & 30 & 30 & 46 & 14 & 50 & 10 & 53 & 07 & 54 & 06 & 60 \\
\hline Ramulu et al $^{7}$ & 47 & 03 & 48 & 02 & 46 & 04 & 43 & 07 & 47 & 03 & 48 & 02 & 46 & 04 & 40 & 10 & 48 & 02 & 44 & 06 & 47 & 03 & 45 & 05 & 50 \\
\hline Pandey et $\mathrm{al}^{8}$ & 83 & 17 & 90 & 10 & 76 & 14 & 90 & 10 & 86 & 14 & 90 & 10 & 88 & 12 & 72 & 28 & 74 & 26 & 95 & 05 & 84 & 16 & 86 & 14 & 100 \\
\hline Present & 100 & 0 & 100 & 0 & 100 & 0 & 95 & 05 & 100 & 0 & 75 & 25 & 98 & 02 & 07 & 93 & 100 & 0 & 23 & 77 & 100 & 0 & 84 & 16 & 100 \\
\hline
\end{tabular}

$A=$ Conventional adequate, $B=$ Conventional inadequate, $C=L D W$ adequate, $D=L D W$ inadequate

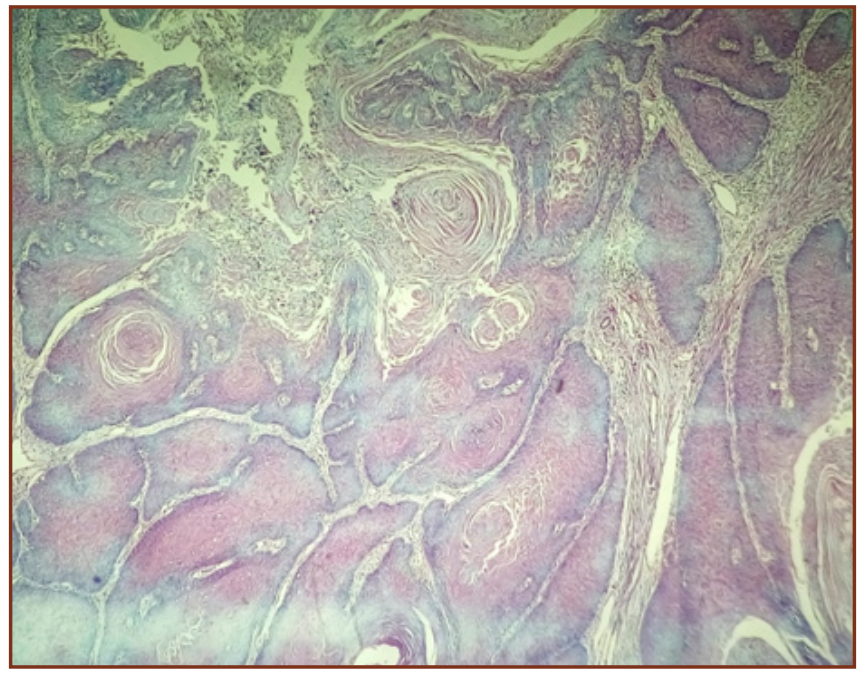

Fig. 1; Section stained following the liquid dish washer protocol showing multiple non uniformly stained areas. H \& E stain, 10X 


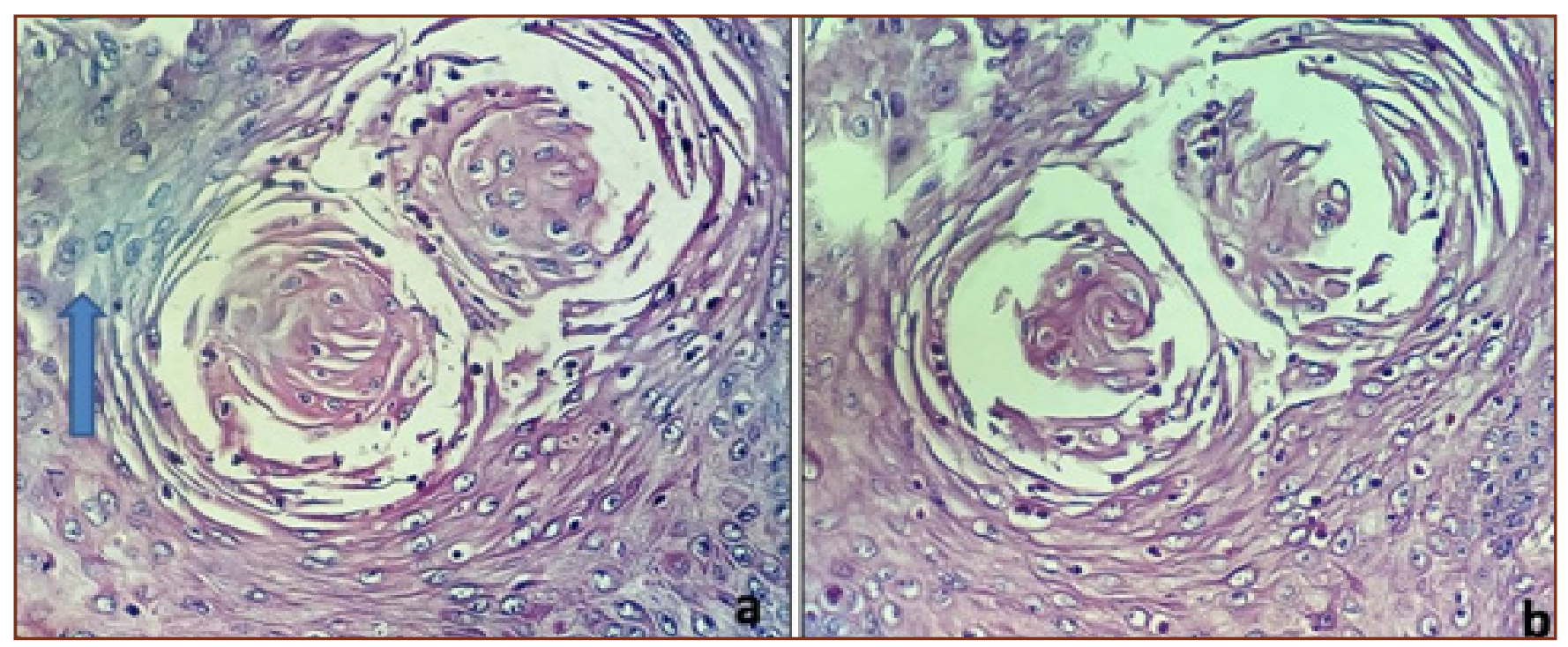

Fig. 2: Section stained following the liquid dish washer protocol (a) showing non uniformly stained area(blue arrow) in comparison with the conventional protocol (b). H \& E stain, 40X.

\section{Discussion}

Routine tissue processing using paraffin wax as an embedding medium requires dehydration, clearing, impregnation and embedding. After the sections are cut with microtomes, sections are dewaxed and hydrated before they are finally stained with Hematoxylin and eosin stains. In this context, xylene forms an inseparable part of a histopathology laboratory. What started as a safe alternative to other harmful and dangerous chemicals like aniline oil, chloroform and toluene, xylene has failed to live up to its expectation of being a safe alternative to these chemicals with proven toxicities noted for long term and short term use of xylene. Although developed nations have stringent guidelines for their usage, in India the monitoring of the usage of such chemicals is slack and lacks standardization. This leads to an indirect impact on the safety of the workers handling these chemicals. In this context, any technique which could minimize or eliminate the use of xylene without compromising on the stain quality or the turnaround time for processing would be much appreciated.

With the harmful effects of xylene being known, many investigators have tried to use substitutes of xylene in histopathology.

Buesa and Peshkov in their study demonstrated that the best clearing agents from both the sectioning quality as well as diagnostic value point of view with automated or manual protocols were 5:1 and 2:1 mixtures of isopropanol and mineral oil. This was followed by undiluted mineral oil on the same parameters. It was observed that the aforementioned solutions performed well at 50 degrees
C. They also noted that prior to staining if sections were dewaxed using a $1.7 \%$ dishwasher solution at 90 degree $\mathrm{C}$ and oven drying the stained sections before coverslipping could help eliminate xylene from the staining tasks. ${ }^{[5]}$

Ankle MR and Joshi PS in their study to evaluate efficacy of xylene free hematoxylin and eosin staining procedure utilized diluted dish washer solution to eliminate xylene and alcohol in their staining protocol. They concluded that xylene and methanol free $\mathrm{H}$ and $\mathrm{E}$ staining was a better alternative compared to the conventional method. In their study they noted that as many as $90 \%$ of sections were stained adequately for offering a diagnosis in the xylene free method compared to $88.33 \%$ in the conventional method. Adequate nuclear staining and better clarity of sections was seen in the xylene free group. But the xylene free group of stained sections scored poorly in uniformity of staining and adequate cytoplasmic staining. ${ }^{[6]}$

Ramulu et al in their study on liquid dish washing soap as a substitute for xylene and alcohol observed that liquid dish washing soap can be used as an alternative and effective substitute to xylene and alcohol in the routine $\mathrm{H}$ and $\mathrm{E}$ staining procedure. They noted that sections stained with xylene free method demonstrated adequate nuclear staining and better clarity of sections compared to the conventional method. They also noted in their study that Harris hematoxylin could be used instead of Meyer's hematoxylin with similar nuclear staining pattern in the xylene free method of staining. ${ }^{[7]}$

Pandey et al in their study to evaluate liquid dish washing soaps as an alternative to xylene and alcohol in hematoxylin 
and eosin staining observed that the soapy sections scored better in the parameters of cytoplasmic staining and crispness of staining. The sections treated with soap were adequate for diagnosis in $86 \%$ of cases. ${ }^{[8]}$

Udonkang et al in their study to evaluate bleached palm oil as a substitute for xylene in histology showed that there were minor differences between the tissues cleared and dewaxed in bleached palm oil at 60 degree $\mathrm{C}$ and xylene in terms of transparency, production of serial sections and quality of histological staining. ${ }^{[9]}$

In an effort in this direction, the current study was undertaken to see if less toxic, cheap and easily available diluted liquid dish washer solution can serve an effective alternative as a deparaffinizing agent for the $\mathrm{H}$ and $\mathrm{E}$ staining method.

The sections stained by both the conventional and the LDW methods were evaluated for the parameters of nuclear staining, cytoplasmic staining, clarity of staining, uniformity of staining and crispness of staining.

On the parameter of nuclear staining, all the sections in both the methods stained well the nuclei. In comparison, other studies too reported low numbers of inadequate cases in the LDW method. (Table 2)

On the adequacy of cytoplasmic staining, the current study showed that almost $95 \%$ of cases showed good cytoplasmic staining. The inadequacies in other studies for the same parameter ranged from $7-17 \%$. (Table 2)

On the issue of clarity of staining, the current study only $75 \%$ of sections showed adequate clarity for satisfactory evaluation in comparison to other studies which had lower levels of inadequacies. (Table 2)

On the issue of uniformity of staining, all the studies which utilized the LDW method had an element of non uniformity of staining. It has been a common observation in all the studies that the xylene free staining procedure is highly sensitive to temperature. The diluted LDW has to be strictly maintained at 90 degree C. A slight drop in the temperature can fail to deparaffinize the sections completely. This leads to microscopic amounts of residual wax on the tissue sections. This can lead to non uniformly stained sections with out of focus areas (Figure 1 and 2) being noted on the slides after staining. Few studies have added an additional step of $1.7 \%$ LDW III at 90 degree $\mathrm{C}$ for $30 \mathrm{~s}$ in their protocol and found better results. This was not done in the present study to note if there were any changes after the addition of a third step of LDW.

The current study showed that fairly most of the sections had crisp quality of staining although other studies have reported a better performance on this parameter. (Table 2)
In the overall scores it was noted that the sections treated with LDW were adequate for diagnosis in $84 \%$ of cases which was similar to other studies. (Table 2)

The statistical analysis suggests that the soapy sections failed to prove that it has comparable effects as that of the conventional method. The $\mathrm{p}$ value obtained was $<0.001$. This is primarily due to the sections performing poorly on the parameters of uniformity of staining and crispness of staining.

\section{Conclusion}

The liquid dish washing solution is composed of non toxic substances which are anionic surfactants. Their concentration in the commercially available dish washer solutions is well monitored by the companies. Using a diluted sample of the sample makes only a meager chance of the product being toxic to the laboratory personnel. The present study shows that LDW can eliminate xylene as a deparaffinizing agent with sections having similar quality of nuclear and cytoplasmic stains although uniformity of staining being an issue to address. It also has added advantages of being non toxic, economical, nonflammable, and non hazardous with no problem of disposal. It also reduces the staining time and is easy to handle.

Modifications to the methodology need to be tried to eliminate the problem of non uniformity of staining and improve the clarity of staining. Also the sections need to be studied after a period of 1 year to see whether the stains have faded.

\section{Acknowledgements}

We acknowledge the technical staff of histopathology section of the department of Pathology, for their help rendered in the conduct of this study.

\section{Funding}

This study was funded by the Indian Council for Medical Research (ICMR), Short Term Studentship for the year 2015

\section{Reference}

1. Stevens A, Wilson I. The haematoxylins and eosin. In: Bancroft JD, Stevens A, editors. Theory and Practice of Histological Techniques. 4th ed., Ch. 6. NewYork: ChurchillLivingstone; 1996. p.99-112.

2. National Institute for Occupational Safety and Health (NIOSH) Occupational health guideline for xylene. Available from http://www.cdc.gov/niosh/docs/81- 123/ pdfs/0668.pdf (Last accessed on 2015 Jan 26)

3. Rajan ST, Malathi N. Health hazards of xylene: a literature review. J Clin Diagn Res. 2014 ;8:271-4.

4. Kandyala R, Raghavendra SP, Rajasekharan ST. Xylene: An overview of its health hazards and preventive measures. J Oral Maxillofac Pathol 2010;14:1-5. 
5. Buesa RJ, Peshkov MV. Histology without xylene. Ann Diagn Pathol 2009;13:246-56.

6. Ankle MR, Joshi PS. A study to evaluate efficacy of xylene free hematoxylin and eosin staining procedures as compared to the conventional hematoxylin and eosin staining. An experimental study. J Oral Maxillofac Pathol 2011; 15:161-7.

7. Ramulu S, Koneru A, Ravikumar S, Sharma P, Ramesh D, Patil R. Liquid dish washing soap : An excellent substitute for xylene and alcohol in hematoxylin and eosin staining procedure. J Orofac Sci 2012; 4:37-42.
8. Pandey P, Dixit A, Tanwar A, Sharma A, Mittal S. A comparative study to evaluate liquid dish washing soap as an alternative to xylene and alcohol in deparaffinization and hematoxylin and eosin staining. J Lab Physician 2014;6:84-90.

9. Udonkang M, Eluwa M, Ekanem T B, Asuquo OR, Akpantah AO. Bleached palm oil as substitute for xylene in histology. Journal of pharmacy and clinical sciences 2014; 8: 8-17. 10.

10 Wikipedia contributors. Dishwashing liquid [Internet]. Wikipedia, The Free Encyclopedia; 2017 Sep 23, 12:47 UTC [cited 2017 Nov 22]. Available from: https://en.wikipedia.org/w/index.php?title=Dishwashing _ liquid\&oldid $=802016653$.

*Corresponding author:

Dr Anikode Subramanian Ramaswamy, 52 Vignesh Nilayam, 11th Cross, T C Palya, K R Puram, Bangalore-560036 INDIA

Phone: +91 09493726494

Email: dr_asr@rediffmail.com 